\title{
Germanica
}

\section{Vérité et sens chez H.-G. Gadamer}

Wahrheit und Sinn Bei H.-G. Gadamer

Jean-Michel Buée

\section{CpenEdition}

Journals

Édition électronique

URL : http://journals.openedition.org/germanica/2440

DOI : 10.4000/germanica.2440

ISSN : 2107-0784

Éditeur

Université de Lille

Édition imprimée

Date de publication : 31 décembre 1990

Pagination : 151-169

ISBN : 9782913857025

ISSN : 0984-2632

Référence électronique

Jean-Michel Buée, «Vérité et sens chez H.-G. Gadamer », Germanica [En ligne], 8| 1990, mis en ligne le 28 novembre 2014, consulté le 06 octobre 2020. URL : http://journals.openedition.org/germanica/

2440 ; DOI : https://doi.org/10.4000/germanica.2440

Ce document a été généré automatiquement le 6 octobre 2020.

(c) Tous droits réservés 


\title{
Vérité et sens chez H.-G. Gadamer
}

\author{
Wahrheit und Sinn Bei H.-G. Gadamer
}

Jean-Michel Buée

1 Dissocier vérité et méthode, c'est d'abord exprimer un refus : celui de réduire la question de la compréhension à sa dimension méthodologique. Comprendre n'est pas un art, ou une méthode, ou une technique. Ce n'est même pas un comportement humain parmi d'autres. C'est l'être de l'être fini, du Dasein, qu'est l'homme. Ou encore c'est une expérience, l'expérience du monde, telle qu'elle s'opère dans le milieu du langage, où homme et monde se rapportent l'un à l'autre en un lien d'appartenance réciproque qui précède toute dissociation entre un sujet et un objet. Gadamer veut ainsi restituer toute son ampleur à ce qu'il nomme «le phénomène herméneutique $»^{1}:$ celuici n'est pas seulement en jeu lorsque nous nous rapportons à notre passé sur le mode des «sciences de l'esprit»; mais aussi bien lorsque nous conversons avec un interlocuteur étranger, ou encore, plus fondamentalement, en cette expérience privilégiée qu'est l'expérience de l'œuvre d'art.

2 La primauté accordée à la méthode n'a fait que dissimuler l'universalité du comprendre. Non du fait de la démarche scientifique elle-même ; mais plutôt à cause de sa présupposition ontologique fondamentale, qui consiste à détruire l'appartenance réciproque de l'homme et du monde, pour ne laisser subsister entre eux qu'un rapport de distanciation aliénante : l'homme se pose comme une conscience, un sujet souverain auquel le réel fait face comme un objet étranger, un simple étant disponible qu'il importe de connaitre, de juger, de manipuler ou d'évaluer à l'aide d'une méthodologie appropriée. La critique du méthodologisme est plus, on le voit, qu'une simple critique du positivisme dominant. Ce qui est en cause, c'est la prétention du Je, de la subjectivité libre à s'ériger en fondement de tout sens et de toute vérité. L'authenticité de l'expérience herméneutique suppose que l'homme cesse de se penser et de se vouloir comme un Je. Elle exige qu'il assume son irréductible finitude, et qu'abandonnant tout désir de maitrise, il se tienne ouvert à la vérité qui s'adresse à lui au travers des paroles, des textes, des œuvres dont la tradition conserve la mémoire. Une mutation décisive en résulte : comprendre ne consiste pas à unifier un divers à l'aide de concepts; mais à écouter la voix d'une tradition, à répondre à l'appel qu'elle nous adresse ; à recevoir 
une vérité que l'histoire nous lègue pour que nous la laissions informer notre présent. Plus que le travail de l'interprète, la compréhension est celui de la chose interprétée (WM ; 441) ; un subir, plus qu'un agir (ibid.), un événement langagier (WM ; 425), non un procès logique.

3 Ces thèmes qui obligent à inverser nos habitudes de pensée, indiquent tous une distance vis à vis de la tradition philosophique occidentale; ou du moins vis à vis de l'identité qui y est posée entre vérité et cohérence discursive. À la prétention de fonder la compréhension et l'entente mutuelles sur la transparence à soi d'un discours absolu, l'herméneutique oppose l'indépassable finitude du Dasein. La vérité n'est pas médiation achevée. Elle n'est pas réponse, toujours assurée, à qui se laisse guider par le travail du concept. Elle est question; une question qui atteint l'être fini au cœur de sa finitude et requiert de lui, qu'au lieu de se laisser fasciner par l'illusion du discours cohérent mais fermé, il soit ouvert, dans le langage, à la vérité des choses qui demande à s'y dire. Cette rupture avec l'idéal du discours absolument cohérent n'entraîne, pourtant, nul abandon de la raison et de l'universalité. L'être fini y trouve au contraire la possibilité d'un rapport au sens qui ne passe pas par le sacrifice de sa finitude : à la raison infinie, mais inaccessible, se substitue l'idée d'une raison finie, ou si l'on préfère d'une raison herméneutique. Le discours et son idéal de maîtrise absolue cèdent la place au langage et à la médiation respectueuse de l'altérité qui s'y opère: l'accord, l'entente, le dialogue, bref l'accès à l'Universel, loin de supposer l'élimination méthodique de nos préjugés et de nos certitudes non réfléchies, ne sont rien d'autre en effet que la structure même de notre être (WM ; 360) : l'ouverture du langage, en laquelle chacun peut dépasser son horizon limité et se rapporter à l'autre, sans pour autant cesser d'être un individu fini et singulier. Ce déplacement, en outre, n'est pas sans conséquences pratiques : il permet de réactiver la question du sens en un monde qui tend à n'envisager sa modernité que sous l'angle de la rationnante technique incarnée par l'expert ou le spécialiste ${ }^{2}$.

4 Il est permis, cependant, de se demander si, à insister sur cette dimension "communicationnelle» ou "pratique », on ne tend pas à effacer quelque peu la spécificité de l'horizon à partir duquel se déploie Vérité et méthode: ne risque-t-on pas de réduire la disjonction qu'énonce le titre à n'être qu'une version renouvelée et affaiblie de la distinction kantienne, ou hégélienne, entre l'entendement et la raison ? ne risque-t-on pas de développer une lecture qui procéderait, elle aussi, de l'horizon même dont l'ouvrage entend dénoncer l'absolutisation: celui de la méthode, de la logique, de la cohérence discursive ? Ces questions nous amènent à privilégier un autre type de problématique. Pour réellement saisir le sens de la thématique du dialogue et de l'entente, il faut, nous semble-t-il, interroger au préalable les transformations de l'idée de vérité qu'entraîne le fait de rompre avec la tradition métaphysique dominante : cet autre rapport à la vérité, cette autre idée de la vérité, à partir desquels l'herméneutique élève sa revendication à l'universalité, quels sont-ils ? La thèse qui, à la fin de l'ouvrage, affirme que «l'être qui peut être compris est langage » (WM : 450) indique manifestement que la vérité cesse de renvoyer à la présence muette d'un ordre éternel offert à la contemplation silencieuse d'une intelligence infinie. Elle devient parlante; ou mieux, elle est parole; une parole vivante, qui surgit comme un appel adressé à chacun, dont la compréhension et l'interprétation, loin d'en être l'image ou le simple reflet appauvri, forment au contraire ce en quoi s'accomplit et se révèle le paradoxe d'un mode d'être dont l'identité suppose la différence de soi à soi que crée le jeu infini de l'interprétation. Ne peut-on faire une hypothèse quant à l'origine de ce 
thème ? Ne renvoie-t-il pas à l'idée d'un jeu de la vérité qui se joue au cœur de la langue et de sa finitude? Ou, si l'on préfère, à l'idée d'une poéticité essentielle du langage, à partir de laquelle serait pensée l'essence de la vérité herméneutique ? Le " phénomène herméneutique " ne désigne-t-il pas finalement, la façon dont s'accomplit, dans le monde de tous et de chacun, ce jeu de la présence et de l'absence, de la dissimulation et de la révélation, du dit et du non-dit, dont la parole poétique est le lieu originaire ? Et, si tel est le cas, ne doit-on pas interroger l'herméneutique sur la prétention à l'universalité ? Celle-ci s'accorde-t-elle avec le fait que la question de la vérité soit posée à partir d'une expérience particulière, dont le caractère éminemment communicable ne suffit pas à effacer la particularité? Si Gadamer délivre l'herméneutique de l'emprise exclusive de la science et de ses procédures objectivantes, n'est-ce pas pour accorder un privilège tout aussi exclusif à la parole poétique, au type de vérité qui s'y montre et au type d'interprétation que celle-ci exige? Ne peut-on prétendre, dès lors, que si l'ouvrage pose bien la question du sens, il le fait de façon réductrice? À privilégier le type de rapport au sens mis en jeu dans le dire poétique, l'herméneutique ne se condamne-t-elle pas à laisser dans l'ombre les autres modalités sous lesquelles l'homme se rapporte à la signification de son monde, en particulier la dimension de l'action, de la praxis qu'elle semble n'aborder qu'avec réticence, d'une manière occasionnelle et abstraite?

\section{Le langage : médiation ou événement?}

$5 \quad$ Les multiples descriptions phénoménologiques que contient Vérité et Méthode semblent de prime abord quelque peu hétérogènes. Elles finissent, pourtant, par apparaître au lecteur comme différentes présentations d'une même thèse fondamentale: celle qu'énonce le dernier chapitre sous la forme de la proposition «l'être qui peut être compris est langage»(WM, 450). Comment entendre cette idée d'une constitution langagière de l'étant susceptible d'être compris? Elle veut d'abord affirmer l'irréductibilité de la chose comprise à la pure objectivité d'un donné, à la facticité d'un immédiat en lui-même dépourvu de sens. Ce qui revient à dire que loin d'être le produit de l'interprétation, le sens en est au contraire la condition : il y a compréhension parce que la chose comprise est signifiante; parce qu'elle est message communicable, parole intelligible, signification potentielle que l'interprétation rend explicite à tous et à chacun, en lui permettant de déployer son pouvoir universalisant : le sens explicité est un sens partagé, un langage commun qui permet aux hommes de dialoguer dans le respect de leurs différences mutuelles. Son être langagier fait de la chose comprise un lien qui rassemble ce qui diffère, la médiation d'une parole vivante qui unit les êtres finis et historiques et les préserve du risque de fermeture et de repli sur soi inhérent à leur finitude. Reste que, si elle ne crée pas le sens, l'interprétation est cependant la condition essentielle de son accomplissement. La chose comprise est acte de signifier et de se signifier, mais ce procès ne devient effectif que par le biais d'une "venue au langage » (WM ; 450) qui est le fait de l'interprétation. Interpréter ne se réduit donc pas à expliciter un sens déjà là, à refléter ou à reproduire une intelligibilité en quelque sorte inhérente aux choses (WM ; $433 ; 446)$. Si la chose est signifiante, elle ne l'est que dans le langage, grâce au langage, qui, seul, en dévoile le pouvoir signifiant et lui permet de s'actualiser. Le langage n'est pas seulement lien qui rassemble; il est aussi venue à l'être de la chose, événement, en lequel surgit sa vérité. L'interprétation est bien transmission et communication d'un sens; mais aussi accomplissement d'une 
vérité, ouverture, où l'acte de l'interprète qui s'ouvre à la chose est en même temps l'acte de la chose qui s'ouvre à elle même, dans l'interprétation, pour venir s'y dire en sa vérité.

La thèse fondamentale de l'ouvrage semble ainsi reposer sur la conjonction de deux thématiques différentes, et même opposées. D'une part, l'affirmation du caractère langagier (Sprachlichkeit) de l'étant évoque l'idée d'une médiation, d'un dialogue entre l'interprète et la chose, où s'instaure un espace commun, source d'un accord et d'une entente qui n'imposent à l'homme aucun reniement de sa finitude. Le thème du langage paraît ici recouvrir celui d'une raison herméneutique, capable de faire communiquer entre eux les multiples mondes humains, sans en réduire la pluralité, ni en effacer l'historicité. D'autre part, la même affirmation évoque l'idée d'un avènement de la vérité dans le langage, d'une ouverture de l'étant, dont l'interprétation est le lieu; ce qui fait d'elle un événement langagier, une rencontre, une expérience, en laquelle le Dasein s'efforce de répondre avec authenticité à l'interpellation d'une parole. Le thème de la Sprachlichkeit semble, cette fois, renvoyer à celui d'une vérité, qui en cette «trace de notre finitude » (WM ; 433) qu'est le langage ne s'ouvre à l'homme que sur le mode du retrait et de la dissimulation. Comment penser ensemble ces deux aspects? Le langage peut-il être à la fois événement et médiation, lieu d'une circulation du sens et lieu d'une venue à l'être de l'étant? Peut-on lui prêter des traits qui en font une sorte d'équivalent fini de l'Esprit absolu hégélien, tout en invoquant une conception de la vérité issue de la déconstruction heideggerienne de la métaphysique?

7 Ces questions suggèrent que l'herméneutique philosophique reposerait sur une équivoque fondamentale ; une sorte d'impossibilité de choisir entre Hegel et Heidegger, entre la tradition métaphysique et sa critique déconstructrice. Pourtant, à bien considérer l'organisation de Vérité et Méthode, il ne semble pas que le discours de Gadamer soit incapable de dépasser cette antinomie; ou du moins d'instaurer un dialogue entre chacun de ses termes. On remarque en effet que le thème du langage ne s'impose pas immédiatement. Il résulte de la description de deux expériences : celle de l'art, puis celle de la compréhension du passé historique, dont la Sprachlichkeit se révèle le fond commun (WM ; 452). Or, chacun de ces deux champs est abordé à partir d'une problématique que l'on peut qualifier d'unilatérale, dans la mesure où elle ne met en avant que l'une des deux déterminations constitutives de la Sprachlichkeit. D'un côté, l'œuvre d'art est posée d'emblée comme le lieu d'un advenir de la vérité, de cette ouverture de l'étant qui est au centre de l'essai de Heidegger sur l'origine de l'œuvre d'art. De l'autre, le problème de la compréhension du passé procède de l'héritage de l'herméneutique $\mathrm{du} \mathrm{XIX}^{\mathrm{e}}$ siècle, où comprendre veut dire instaurer un lien interpersonnel, une médiation par laquelle tout individu peut communiquer avec tout autre, aussi étranger qu'il soit, en se projetant dans son intériorité ou en déchiffrant les signes objectivés d'une vie que l'interprétation doit ressusciter en son altérité. Si ces remarques sont correctes, il faut alors supposer que Vérité et Méthode entend rectifier ce que ces deux perspectives ont d'unilatéral. Et ce, en révélant au cœur de chacune d'elles la présence agissante de l'autre, de celle qu'elle semble devoir exclure. Ainsi, dans la première partie s'agirait-il de prévenir toute dérive irrationnaliste en montrant que l'advenir de la vérité dont l'art est le lieu s'accomplit comme une médiation, en laquelle communiquent des mondes humains que sépare leur distance temporelle. La seconde partie, à l'inverse, viserait à rappeler la finitude de notre être, en pensant la médiation du passé et du présent comme l'ouverture d'une tradition à sa propre 
efficience historique; comme l'advenir d'une parole de vérité, qui, telle une œuvre d'art, ne cesse, en se renouvelant, de renouveler notre rapport au monde.

\section{L'œuvre d'art : la vérité comme vérité interprétée}

Le détail du texte permet-il d'y discerner l'existence de ce double mouvement, qui ne nous mène de la vérité au sens que pour nous ramener du sens à la vérité ? Tentons d'abord de mettre en évidence le premier point. Quel est, l'horizon à partir duquel se déploie la critique de la conscience esthétique? Il s'agit, de toute évidence, du questionnement ontologique hérité de Heidegger. Encore faut-il noter que l'idée d'un advenir de la vérité n'est jamais énoncée en tant que telle. Elle est plutôt la présupposition de l'analyse, évoquée indirectement, notamment à travers le concept d'une "transmutation en figure " (Verwandlung ins Gebilde) par laquelle le jeu humain s'accomplit comme art (W; 105). L'idée est celle d'une mutation brusque, d'une métamorphose par laquelle une chose, subitement, devient autre. Or, cette métamorphose n'est pas enchantement, ensorcellement magique qui détourne du réel ; mais au contraire, retour à l'être vrai, mise en suspens d'une réalité quotidienne que le monde de l'œuvre éclipse pour s'imposer avec une plénitude et une présence telles que «face à lui, chacun reconnaît : c'est bien ainsi qu'il en est » (WM ; 108). Abolition de la réalité familière, « suppression qui l'élève à sa vérité » (ibid.), l'art est bien comme chez Heidegger événement de vérité. En l'œuvre s'ouvre un monde, qui n'est ni produit illusoire de notre imagination ni copie appauvrie du réel, mais ce réel, lui-même « dans la vérité plus intense de son être " (WM ; 130). Reste, cependant, que cette thématique s'organise à partir d'un concept que la méditation heideggérienne récuserait sans doute comme "métaphysique»: le concept de Darstellung (WM; 98), d'exhibition ou de présentation. La venue à l'être de la vérité est une présentation de soi-même. Ce qui signifie que l'œuvre n'est pas le produit à jamais figé d'une création singulière ; mais plutôt le jeu sans cesse renouvelé de ses multiples interprétations, un perpétuel différer où, paradoxalement s'accomplit une identité. L'analyse prend, ici, pour fil conducteur, les « arts transitoires » (WM ; 130), tels la musique ou le théâtre, où chaque nouvelle représentation actualise une nouvelle potentialité de sens, qui enrichit l'œuvre sans en détruire l'unité. Mais l'examen des autres champs esthétiques permet d'effacer la particularité de ce point de départ: la Selbstdarstellung est bien la constitution ontologique de toute œuvre d'art, celle de l'image picturale, du monument ou du texte littéraire autant que de la tragédie ou de la pièce musicale. Que signifie ce changement de problématique par rapport à Heidegger ? Pourquoi l'œuvre cesse-t-elle d'être «combat du monde et de la terre $»^{3}$, tension de l'ouverture et de son retrait? Pourquoi devient-elle unité de la vérité et de sa présentation?

Dénoncer l'illusion d'un être en soi, distinct des circonstances dans lesquelles il surgit, c'est indiquer que la rencontre d'un «public » est essentielle à l'œuvre, car elle est ce par quoi s'accomplit sa vérité. Autrement dit, l'avènement de la vérité est "médiation », par laquelle l'œuvre " accède à son être authentique » (WM ; 112). Cette médiation est l'historicité même de l'œuvre, la temporalité qui lui est inhérente comme à tout étant dont l'être n'est qu'un incessant devenir autre (WM ; 117). Mais, loin d'indiquer une perte ou une destruction, cette temporalité signifie au contraire un surplus d'être. L'œuvre d'art, en effet, peut-être caractérisée par sa « contemporanéité » (WM ; 115), qui n'est pas la simultanéité d'un rapport entre 
consciences. Mais une médiation totale, une sorte de « présent intemporel » $(\mathrm{WM} ; 272)^{4}$ où s'atteste l'efficience d'une vérité capable d'imposer sa présence en chacun des multiples horizons historiques qui l'accueillent. La vérité de l'art s'accomplit donc comme la continuité et la permanence d'une histoire. Elle est l'incessante recréation d'un sens dont la présence n'est effective que dans la médiation qui fait communiquer entre eux passé et présent, monde de l'œuvre et monde du spectateur, du lecteur ou de l'auditeur.

10 Il nous semble que cette analyse procède pour l'essentiel de la réintroduction d'éléments « hégéliens" au sein d'une problématique de type heideggerien ${ }^{5}$. La vérité est l'ouverture d'un monde; mais qui s'accomplit comme présentation renouvelée de soi ; autrement dit comme un événement dont la finitude n'est plus réserve et retrait, mais histoire, ré-effectuation constante d'une vérité qui se fait valoir comme la contemporanéité d'un pur présent, capable de réunir les horizons historiques les plus éloignés. La vérité est bien événement; mais comme l'événement d'une présence, qui s'actualise sous la forme d'une médiation, comme "cette médiation du passé et du présent " qui advient en toute œuvre d'art (WM ; 149). Le thème de la médiation n'annule pas l'idée de l'ouverture ; il vient simplement rectifier ce que celle-ci pouvait avoir d'unilatéral. Correctif qui permet de penser l'être de l'œuvre d'art comme être herméneutique. C'est-à-dire comme avènement d'une vérité qui est langage, communication d'un sens à comprendre et à interpréter. La question de l'œuvre d'art est soustraite à l'esthétique et à son subjectivisme; mais c'est pour être reprise dans une herméneutique, qu'il convient dès lors de redéfinir et d'élargir: «la compréhension doit être pensée comme une partie de l'événement du sens, en lequel se forme et s'accomplit le sens de toute énonciation - celle de l'art et celle de tout autre type de communication» (WM ; 157). Ainsi énoncée, la tâche apparait bien comme inverse de celle du début. S'il s'agissait d'abord de penser l'événement de vérité qu'est l'art comme communication d'un sens, il s'agit cette fois de penser la communication comme avènement d'une vérité.

\section{L'histoire : l'interprétation comme écoute de la vérité}

11 Toute entreprise herméneutique, l'étymologie l'indique, procède d'un «esprit de médiation» (WM ; 157). Encore faut-il en préciser la signification : s'agit-il d'éliminer les préjugés, dont l'étroitesse, en restreignant notre horizon, lui interdit de s'ouvrir à l'altérité ? S'agit-il au contraire d'en partir, en tant qu'ils sont préjugés, c'est-à-dire horizon de sens qui précède tout jugement et toute réflexion? Le choix de la seconde perspective n'est pas la simple répétition de la réaction romantique contre l'Aufklärung. Il s'agit plutôt de lutter contre le méthodologisme inhérent à toute problématique de la subjectivité, à partir d'une analytique du Dasein. Regarder le passé comme un objet, c'est oublier en effet que la question de sa compréhension se pose à l'être fini, inséré dans un monde qui le précède et le détermine. Autrement dit, c'est oublier que l'interprète se meut d'emblée dans un cercle, où le passé se détache sur le fond d'un présent éclairé en retour par ce passé dont il est l'aboutissement : l'historien est luimême un être historique, dont le rapport à l'histoire n'est pas de l'ordre d'une maîtrise, mais d'une appartenance. En ce sens, le préjugé n'est pas isolement et repli sur soi, mais ouverture. Il témoigne de notre lien au passé, comme écho d'une parole qui continue à résonner dans la nouveauté du monde qu'elle a contribué à façonner. Trace, 
où s'indique la continuité d'une tradition, le préjugé est ainsi le point de départ de la compréhension: une anticipation du sens, une pré-compréhension d'où peut se déployer un regard sur le passé. Encore faut-il qu'une telle anticipation soit légitime. Mais cette légitimation ne saurait être fournie par la réflexion. Elle provient plutôt de la tradition elle-même; ou, plus exactement, de l'authenticité avec laquelle nous répondons à l'appel qu'elle nous adresse. Autrement dit, le passé n'est pas un passé mort; mais une parole vivante qu'il convient d'accueillir et d'entendre comme une parole de vérité. Parce qu'elle est tradition qui ne cesse d'informer notre présent, l'histoire n'est pas la transmission d'un sens auquel nous serions libres de demeurer indifférents; elle est avènement d'une vérité qui doit transformer notre rapport au monde. Cela ne signifie pas que la compréhension doive consister à revivre le passé, en une immédiateté qui annulerait l'histoire : il y a une altérité du passé, qui impose la tâche d'une médiation, d'un dialogue en lequel l'horizon propre de la chose doit venir se fondre avec celui de l'interprète (WM ; 289). Mais, malgré le vocabulaire phénoménologique, cette fusion n'est pas rapport de deux consciences : si le rapport au passé possède bien l'aspect d'un lien entre un Je et un $\mathrm{Tu}$, ce qui importe dans cette relation, ce n'est pas l'autre ; c'est ce qu'il dit (WM ; 341 sq). La différence et la distance existent, mais sur le fond d'une entente préalable, d'une identité qui n'est autre que la permanence de l'histoire. Le dialogue n'est pas tant dialogue avec la tradition que dialogue de celle-ci avec elle-même. L'ouverture à l'autre est finalement ouverture à soi de la vérité, événement, en lequel la vérité se rapporte à sa propre efficience historique. Si l'interprétation est prise de conscience d'un travail du sens, celui-ci n'est pas l'œuvre de l'interprète; mais celui de la chose interprétée, dont la vérité se renouvelle en renouvelant chacun des horizons historiques qu'elle pénètre: la conscience historique est une Wirkungsgeschichtliches Bewusstsein (WM ; 284 sq ; 324 ; 329) qui est « plus être que conscience » (mehr Sein als Bewusstsein)6.

Les sciences de l'esprit ont bien affaire au problème de la communication et de la médiation, du dialogue, d'où doit naître l'entente. Mais celle-ci ne résulte pas de l'adaptation mutuelle de deux sujets. Elle est l'avènement d'une vérité qui, toujours déjà réunit ce que la distance temporelle sépare. Aussi la médiation historique est elle rencontre du vrai, expérience authentique (WM ; 329 sq.), qui n'est pas manifestation d'une cohérence absolue, mais événement de langage. Il y a bien une «dialectique » de l'interprétation; mais celle-ci n'est pas l'infinité d'un parcours qui mènerait à l'achèvement d'une réponse. Elle est le renouvellement d'une question ouverte, qui est plus celle du texte interprété que celle de l'interprète (WM ; 344 sq. ; 441 sq.). Acte de l'être fini, l'interprétation est une "écoute" (hören) (WM ; 438) où se fait valoir la présence irrécusable de la chose, et non le choix libre d'un sujet guidé par ses intérêts (WM ; 439). Le langage est bien communication d'un sens; mais seulement dans la mesure où surgit, dans l'historicité même de l'expérience, la vérité de la tradition qui la soutient : la médiation langagière n'instaure un lien entre le passé et le présent que pour y laisser advenir les paroles de vérité qui habitent notre histoire.

Si la première partie s'efforçait de réintroduire la thématique de la communication dans celle de l'ouverture, la seconde entend au contraire saisir la médiation historique comme l'ouverture à soi par laquelle se signifie la vérité d'une tradition. Cette fois, ce sont des concepts de type hégélien qui sont repensés à la lumière d'une problématique de la finitude interdisant à l'expérience de l'histoire de jamais se résoudre en l'achèvement d'un savoir de soi (WM ; 285). 


\section{Logos et poïesis}

14 Le statut que l'herméneutique confère au langage résulte, nous semble-t-il, de ce double mouvement; qui dit à la fois le refus de réduire la vérité à la singularité d'un événement indicible, et celui de réduire le sens à la transparence à soi d'une cohérence absolue où cesserait de parler la finitude humaine. Le langage n'est pas ouverture de l'étant ou communication. Il est l'un et l'autre, médiation et événement, vérité qui est sens, sens qui est vérité. Cette "synthèse » unit deux horizons qui paraissent pourtant impossibles à unir : celui pour lequel le langage est avant tout poïesis, où l'étant se dévoile en sa vérité; et celui pour lequel il est avant tout logos, raison dont l'universalité rassemble ce qui semble séparé. Peut-il s'agit d'autre chose que d'un éclectisme, résultant de la simple addition d'éléments hétérogènes? Il nous semble que la visée de l'herméneutique est bien de dépasser ce qui ne serait qu'une apparence de synthèse. Et ce, dans la mesure où elle tente de penser à la fois la médiation comme poïesis et la poïesis comme médiation.

15 Le premier point se dégage du concept même de Wirkungsgeschichte (WM ; 284). Comme l'a montré J. Grondin7, l'idée est celle d'un « travail de l'histoire » par lequel la vérité transmise se fait valoir comme un sens que renouvelle chacune de ses rencontres avec un horizon historique différent. Or cette médiation suppose un jeu créateur. Comment en effet le sens pourrait-il circuler d'un horizon à l'autre si la traduction qu'est toute interprétation n'était pas une "recréation " (Nachbildung) (WM ; $363 ; 365 ; 448)$ ? Non au sens d'une création seconde, qui imposerait à la chose l'arbitraire de l'interprète (WM ; 377). Mais au sens d'une nouvelle présentation où la chose s'assimile la nouveauté du contexte en lequel elle pénètre. Ni simple redite, ni simple reproduction, l'interprétation est "comme une nouvelle création opérée par la compréhension " (WM ; 448), un acte « poïetique » qui « dans des mots apparemment usés et hors d'usage sait éveiller une vie secrète " (WM ; 426) : parce qu'elle est événement langagier, la médiation historique est un événement créateur par lequel "quelque chose surgit, quelque chose existe désormais qui n'existait pas avant» (WM ; 438). Le langage est universalité et raison (WM ; $379 ; 380 ; 452)$; mais seulement comme travail, « œuvre » (WM ; 359), dont la «métaphorique fondamentale» (WM ; 406) permet de restituer cette autre poïesis qu'est le travail de l'histoire.

Il n'en résulte nul abandon de l'idée d'une vérité commune et partagée. Ainsi l'analyse de l'expérience esthétique ne cesse-t-elle de récuser le thème de la génialité ou celui de l'Erlebnis pour insister sur le pouvoir universalisant d'un langage qui s'adresse à chacun ${ }^{8}$. Qu'il s'agisse de l'art ou de l'histoire, l'expérience herméneutique est une expérience qui nous transforme. Non en nous détournant du réel, comme le ferait une opération magique. Mais en "refigurant ${ }^{9}$ notre présent, c'est-à-dire en enrichissant l'unité qui fait de lui un monde, et en contribuant ainsi à l'orientation concrète de l'individu. Tel paraît être le sens de la reprise de l'ancienne problématique herméneutique de l'« application » (WM ; 209 sq.). Qu'il s'agisse d'une œuvre d'art, d'un ensemble d'événements, d'un texte ou d'une règle juridique, le sens n'est jamais un fait ; mais un procès où s'affirme la validité d'une tradition, dont la réception n'est pas séparable de sa concrétisation: l'interprétation doit éclairer le présent; elle doit permettre à chacun de s'y mouvoir plus aisément en lui offrant une sorte de « sagesse pratique ", comparable à la phronesis aristotélicienne, où l'universel de la règle ne vaut 
que dans la singularité des situations concrètes ${ }^{10}$. Même s'il présente de manière plus intense les traits qui font du langage un événement (WM ; 445), le verbe poétique n'est que «l'intensification » du "discours de tous les jours» (WM ; 446). Aussi est-ce dans cette quotidienneté que doit s'inscrire la vérité qui, dans l'interprétation, vient à l'être : la puissance poétique du langage trouve son accomplissement dans une " refiguration " du monde, qui y accroît les possibilités de dialogue et de communication mutuelle.

Cette «compénétration » de la poïesis et de la raison traduit un double refus : celui d'une raison dont le pouvoir critique ne s'ancrerait pas dans la couche de sens antérieure à la réflexion qu'est le monde vivant et vécu de la tradition ${ }^{11}$. Mais aussi celui de toute restauration romantique qui, en-deçà du logos, prétendrait en revenir au mythos, en valorisant l'ancien seulement parce qu'il est ancien (WM ; 257). Reste, cependant, qu'il est deux façons de concevoir une telle articulation: à partir de la raison; dont la dimension " créatrice » se signale par une capacité à fonder et à guider la praxis historique de l'homme ${ }^{12}$. À partir de la poïesis, dont la dimension « raisonnable » résulte d'un pouvoir de refiguration qui lui permet de "proposer » à tous et à chacun un monde habitable où communiquent les multiples expressions d'un sens commun que temps et espace tendent toujours à fragmenter. Le geste fondamental de l'herméneutique est manifestement plus proche de la seconde de ces positions. Aussi paraît-elle, finalement, accorder un privilège à la dimension "poïetique » du langage. C'est ce qui résulte du thème d'une "métaphorique fondamentale " que nous avons déjà évoqué (WM ; 406). Mais aussi et surtout de l'idée d'une "structure spéculative du langage » (WM ; 432 sq.), mise en évidence à partir d'une référence au verbe poétique, et notamment à la parole de Hölderlin selon laquelle « il importe que le poète ne tienne rien pour donné, qu'il ne parte de rien de positif, que la Nature et l'Art, tels qu'il les a appris auparavant et les voit à présent, ne parlent pas, avant que pour lui, n'advienne un langage » (WM ; 446) ${ }^{13}$. La dimension de la vérité prend ici manifestement le pas sur celle du sens. Le langage n'est jamais une «forme » que l'on pourrait isoler du contenu transmis (WM ; 381; $382 ; 416 ; 417)$, il ne se réduit pas à l'abstraction d'une structure qui articule et met en relation; mais il est l'événement spéculatif d'une parole qui " porte chaque chose à être ${ }^{14}$ et nous la rend présente, en "reflétant » une relation à l'ensemble de l'être. En celui qui parle, celle-ci vient au langage (WM; 445 sq.); autrement dit, dans le mot, la vérité surgit, comme une "lumière " soudaine, qui élargit notre expérience en l'éclairant d'un jour nouveau. Cet événement est une manifestation de soi, une présentation, dont la finitude n'empêche nullement la vérité d'« être là », en une présence qui, telle l'Idée platonicienne du Beau, abolit toute distance entre l'être et son apparaître (WM ; 452 sq.). Cela n'implique pas qu'il y aurait une interprétation ultime, où la vérité de la chose serait définitivement révélée; mais seulement que cette vérité, dont l'être est un perpétuel renaître, est toujours là, comme un « jeu» qui ne cesse de jouer et de se jouer au cœur du langage : « le jeu du langage lui-même, qui s'adresse à nous, qui propose et retire, qui interroge et s'accomplit luimême dans la réponse » $(\mathrm{WM} ; 464)$.

\section{Herméneutique, christianisme et liberté}

Le privilège de la poïesis ne peut coexister avec la reconnaissance du logos et de la raison qu'à une condition : découvrir un langage commun qui soit à même d'exprimer les deux dimensions. Ce langage, l'herméneutique le trouve, nous semble-t-il, dans 
l'héritage religieux, et plus précisément, dans une exploitation de la thématique centrale du christianisme, celle de l'Incarnation ${ }^{15}$. Quoiqu'en dise Gadamer (WM, Préface XVII), il semble en effet que, plus encore que l'œuvre d'art, ce soit l'événement christique qui fournisse le paradigme de la vérité herméneutique. Pour le croyant, c'est là l'événement par excellence, celui où advient la vérité; et, qui plus est, une vérité qui est parole, verbe incarné. De plus, il s'agit d'un événement fondateur, qui rassemble les fidèles en instituant entre eux le lien médiateur d'une communauté. En outre, même si elle a eu lieu " une fois pour toutes ", l'Incarnation ne cesse de s'accomplir et de se renouveler à travers le culte et la prédication dont le rôle est d'en manifester la vérité aux yeux de tous. Comment ne pas remarquer, enfin, que nombre de thèmes essentiels de Vérité et méthode, tels la tradition, l'appe ${ }^{16}$, l'écoute ${ }^{17}$, la rencontre, et même le dialogue ou l'entente, sans être spécifiquement chrétiens, correspondent cependant à certains des traits essentiels de tout christianisme?

Que l'Incarnation fournisse à l'herméneutique le modèle même de toute vérité, ou qu'elle ouvre simplement un champ particulier d'interprétation, une chose, du moins, semble claire : c'est le rejet radical de toute problématique pour laquelle le sens serait une simple création humaine. S'il est le lieu du sens, le langage ne l'est que dans la mesure où s'y déploie le jeu créateur d'une vérité ; jamais celui d'une liberté. Ce point dérive manifestement du statut accordé à la subjectivité, en laquelle l'herméneutique ne voit qu'une volonté de pouvoir, de maîtrise, de domination, de manipulation, origine d'un méthodologisme dont il convient de démasquer et de combattre toutes les formes $(\mathrm{WM}, \text { Préface XXIII, } 426 \text { sq. })^{18}$. Il est difficile de penser qu'il y ait là autre chose qu'une conception réductrice, héritée de la lecture heideggerienne de l'histoire de la

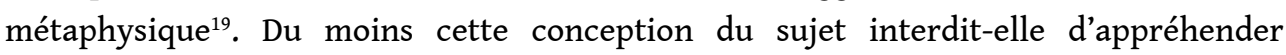
l'histoire comme le domaine où "l'homme n'aurait affaire qu'à lui-même et à ses propres créations » $(\mathrm{WM} ; 260,355)$. Ce qui exclut non seulement l'idée d'une médiation absolue (WM ; 324 sq.), mais aussi celle d'un sens dont l'histoire universelle serait ou pourrait être la réalisation (WM; Préface XXI, 354): l'histoire est un destin, qui demeure étranger à l'homme, au même titre que la nature, même si c'est d'une autre façon $(\mathrm{WM} ; 260)^{20}$. Comment dès lors penser l'action, ou si l'on préfère, la politique? Même si la problématique de l'application tente d'apporter une réponse, celle-ci semble trop générale pour être satisfaisante : comment en effet faire entrer positivement le champ de la société et de l'État dans l'horizon d'une problématique pour laquelle le principe de la liberté et de l'autonomie, qu'il débouche sur une praxis effective ou sur un rapport critique de la pensée au réel, n'est jamais qu'un préjugé illégitime (WM ; 26159) témoignant de l'oubli du Dasein et de sa finitude?

Nous nous étions demandé si l'herméneutique de Gadamer ne reposait pas sur un privilège accordé à la poïesis; et si cela ne venait pas, dans une certaine mesure, en limiter l'universalité. Dans les deux cas, il semble que la réponse puisse être largement positive. En concevant le sens comme l'avènement d'une vérité, et non comme le produit d'une libre spontanéité, l'herméneutique valorise l'ouverture poïetique et se condamne à laisser au second plan la critique et la transformation de ce qui est ${ }^{21}$. Reste pourtant que la poéticité du langage n'exclut nullement sa capacité à unir les hommes. Le langage est poïesis, mais une poïesis qui s'accomplit comme raison : invoquer le langage ne veut pas dire, ici, éliminer le concept et le discours. Ce que refuse l'herméneutique, c'est seulement l'idée d'une autonomie et d'une cohérence propres de la logique du sens : la formation du concept est une «formation naturelle» (WM ; 405) 
qui est depuis toujours à l'œuvre dans le langage, comme l'avait vu Aristote (WM ; 408 ; 409). Ainsi l'accès à la cohérence ne repose-t-il jamais sur un choix libre, sur un acte de liberté (WM : 439, 465) qui, détachant le discours de son fond langagier, lui permettrait d'acquérir une autonomie, absolue ou relative ${ }^{22}$ : la dimension de la raison est inhérente au langage lui-même, dont la puissance de révélation poétique suffit à fonder la possibilité de la médiation et du dialogue. On peut toutefois se demander si le fait même du discours de Gadamer ne vient pas contredire une telle position. La raison qui décrit l'expérience herméneutique participe-t-elle encore de l'expérience qu'elle décrit ? Le rapport à la vérité qui sous-tend toute l'entreprise demeure-t-il un rapport herméneutique? L'idée même d'une herméneutique philosophique n'implique-t-elle pas une autre idée de la philosophie que celle qui en fait l'explicitation de l'être herméneutique de tout étant compréhensible? Ne suppose-t-elle pas aussi une réflexion sur l'interprétation, un discours auquel l'être langagier des choses peut se montrer dans la mesure où il s'en sait séparé par la distance que crée la réflexion? Au fond de cette ambiguïté fondamentale, consistant à mettre en question le primat du concept au sein d'un langage qui reste lui-même conceptuel, ne voit-on pas resurgir la dualité que l'herméneutique entendait dépasser : celle de la tradition métaphysique et de sa critique déconstructrice? ?23.

\section{NOTES}

1. - H.G. Gadamer, Wahrheit und Methode (W.M), Tübingen, J.C.B. Mohr, 1960, 2. Auflage, 1965, p. XXVI. trad. française partielle Vérité et Méthode, Paris, éd. Du Seuil, 1976, p. 21. Nous citerons désormais la pagination de l'édition allemande (WM) puisqu'on la retrouve en marge de l'édition française.

2. - C'est le thème que développe l'essai plus récent, Vernunft im Zeitalter der Wissenschaft, Frankfurt, a.M., Suhrkamp, 1976.

3. - M. Heidegger, Der Ursprung des Kunstwerkes in Holzwege, Frankfurt a.M., Klostermann, 1949. trad. française L'Origine de l'œuvre d'art in Chemins qui ne mènent nulle part, Paris, Gallimard, 1962, p. 53.

4. - Cf. aussi l'article de 1964, Ästhetik und Hermeneutik in Kleine Schriften, Tubingen, J.C.B. Mohr, 1967-1977 où Gadamer déclare que « l'œuvre d'art occupe un présent intemporel ».

5. - C'est ce que montre par ex. G. Vattimo dans l'introduction à sa traduction italienne de Wahrheit und Methode. Introduzione, l'ontologia hermeneutica nella filosofia contemporanea, Milano, Fabbri, 1972, p. VIII-XVI.

6. - Kleine Schriften I, p. 127, 158.

7. - Dans un article intitulé «La conscience du travail de l'histoire et le problème de la vérité en herméneutique», in Archives de philosophie, $\mathrm{n}^{\circ} 44$, juillet-septembre 81, Paris, Beauchesne. J. Grondin ne propose pas seulement de traduire Wirkungsgeschichte par «travail de l'histoire ». Il montre que, conçue comme travail, la vérité est une vérité poïetique, une «alétheia poiétiké».

8. - Cf. in WM ch. 1, «Le dépassement de la dimension esthétique » le passage sur "l'esthétique du génie et le concept d'Erlebnis ». Cf. aussi l'article cité dans la note 4, où Gadamer écrit : «le langage de l'art est constitué précisément par le fait qu'il s'adresse à l'auto-compréhension de 
chacun». Cf. enfin la conférence «Die Universalität des hermeneutischen Problems», Kleine Schriften I, trad. française in L'Art de comprendre, Paris, Aubier, 1982, p. 29.

9. - Le concept se trouve chez P. Ricœur plus que chez Gadamer. Cf. Temps et Récit, Paris, éd. du Seuil, tome 1, p. 109 sq. Mais outre le fait que Ricœur fasse référence à Gadamer (cf. p. 109 et p. 122 note 1), l'idée gadamérienne de Nachbildung est bien celle d'une "refiguration ", issue comme chez Ricœur d'une réinterprétation de la mimésis aristotélicienne.

10. - Cf. aussi le texte intitulé Hermeneutik als praktische Philosophie in Vernunft im Zeitalter der Wissenschaft, p. 108 où Gadamer écrit «l'herméneutique est philosophie, et, en tant que philosophie, elle est philosophie pratique».

11. - Cf. WM 266 sq., cf. aussi le débat avec Habermas dont on trouvera les éléments essentiels en français dans l'ouvrage d'Habermas, Logique des sciences sociales et autres essais, Paris, PUF, 1987, p. 184-215 et p. 239-273 et dans le recueil de textes de Gadamer traduits sous le titre L'Art de comprendre, p. 123-174.

12. - Telle est, par exemple la position d'Éric Weil dont la Logique de la philosophie (Paris, Vrin, 1950) pense la catégorie de l'Action comme le dépassement de l'antinomie de la raison (de l' Absolu) et de la poïesis (du Fini), en une synthèse pratique qui constitue l'ouverture même de l'histoire, dont le Sens, toujours à réaliser, est l'œuvre de la liberté raisonnable de l'être fini. Sur ce point, cf. le livre de G. Kirscher, La Philosophie d'Éric Weil, Paris, PUF, 1989, p. 327 sq.

13. - Cf. aussi ce qui est dit de la parole poétique dans la Postface (1972) de WM. trad. française in L'Art de comprendre, p. 114-116.

14. - Cf. in WM, 464 la référence au poème de $\mathrm{S}$. George, Le Mot et à son dernier vers "Aucune chose ne soit, là où le mot faillit ». Gadamer veut ici, de toute évidence, faire allusion aux textes de Heidegger Das Wesen der Sprache (trad. française «Le déploiement de la parole ») et Das Wort (trad. française «Le mot») réunis in Unterwegs zur Sprache, Pfullingen, G. Neske, 1959, trad. française Acheminement vers la parole, Paris, Gallimard, 1976. Dans le premier de ces textes, Heidegger commente le vers de George en disant «le mot... porte chaque chose à être et le comporte en l'être » (trad. Française p. 161).

15. - Cf. WM 395 sq., ainsi que 405, où Gadamer écrit : « Ce que nous avons nommé expérience herméneutique trouvera ici (dans la christologie) son véritable fondement ». Outre la thématique de l'Incarnation, il faut aussi signaler le rôle important que joue la référence à Platon et au fond religieux de la notion de theoria. Cf. WM. 118 sq., en particulier la note 2, p. 118.

16. - Cf. WM 120 et la référence à la réflexion théologique de Kierkegaard.

17. - Cf. les remarques sur le thème de l'écoute dans la tradition vétéro-textamentaire dans l'article sur « Hegel et Heidegger » in Hegels Dialektik, Tubingen, JCB Mohr, 1980 ; trad. française in L'Art de Comprendre, p. 193. Pour l'influence de Heidegger sur la théologie chrétienne, p. 99-112, cf. aussi l'appendice à WM (écrit de 1972) intitulé Hermeneutik und Historismus trad. française in L'Art de comprendre, p. 66 sq.

18. - Cf. aussi la conférence citée dans la note 8 où Gadamer parle « des lois fondamentales de notre être soustraites à notre arbitraire et qu'il ne nous appartient plus de faire mais d'honorer ", trad. française in L'Art de comprendre, p. 28.

19. - Cf. par ex. les critiques de Cassirer à l'égard du Kantbuch de Heidegger ; en français Débat sur le kantisme et la philosophie (Davos, mars 1929) et autres textes de 1929-1931, Beauchesne, Paris, 1973. Cf. aussi, sur ce point, le livre de A. Renaut, L'Ère de l'individu, Paris, Gallimard, 1989.

20. - Cf. aussi « À propos de l'herméneutique » in L'Art de comprendre, p. 42-43.

21. - Ce point est d'ailleurs explicitement reconnu par Gadamer lui-même, cf. WM, Préface, p. XXIII.

22. - La première position correspondrait par exemple à la Logique de Hegel, cf. Die Idee der Hegeischen Logik in Hegels Dialektik, p. 65-85. Trad. française in L'Art de comprendre, p. 201-223. La seconde à la Logique de la philosophie d'Éric Weil où le discours ne se détache du langage que pour 
l'apercevoir au terme du parcours de ses catégories, comme la forme infinie du Sens qui permet de les articuler sans les réduire.

23. - C'est aussi ce que semble indiquer la coexistence de deux types de critiques, radicalement inverses l'une de l'autre. D'un côté, celles qui reprochent à l'herméneutique l'imprécision de ses concepts ; une imprécision qu'elle revendique en y voyant la marque d'une « détresse du langage, innée dans la philosophie ", et dissimulée par «l'harmonie des systématiques conceptuelles ", cf. WM Postface (1972), trad. française in L'Art de comprendre, p. 102. De l'autre des critiques qui, telle celle de J. Derrida, reprochent au dialogue et à l'expérience herméneutiques de rester prisonniers de la clôture logocentrique, ne serait-ce que parce que «souvent les métaphysiques (et peut-être même toutes) se sont présentées comme les descriptions de l'expérience même, de la présentation même ». Cf. Text und Interpretation Eine deutsch-französische Debatte mit Beiträgen von J. Derrida, Ph. Forget, M. Frank, H.G. Gadamer, J. Greisch, F. Lamelle, München, W. Fink Verlag, 1984. On trouvera la conférence de Gadamer, la critique de Derrida, et la réponse de Gadamer, en français, dans la Revue internationale de philosophie, $\mathrm{n}^{\circ} 151,1984$.

\section{RÉSUMÉS}

En affirmant que «l'être qui peut être compris est langage ", l'herméneutique philosophique de H.-G. Gadamer semble hésiter entre deux directions: celle qui, à la suite de Hegel, pense le langage comme logos, c'est-à-dire comme médiation, communication et circulation d'un sens, et celle qui, à suite de Heidegger le pense comme poïesis, c'est-à-dire comme ouverture de l'étant, venue à l'être de la vérité. On s'interroge sur la façon dont Vérité et Méthode dépasse cette antinomie. Le langage est à la fois ouverture et médiation, logos et poïesis, vérité qui est sens, sens qui est vérité. Mais, en cette unité, la dimension privilégiée n'est-elle pas la poïesis, dans la mesure où le jeu qui se joue dans le langage est toujours celui de la vérité, jamais celui de la liberté? Son refus du subjectivisme permet-il dès lors à l'herméneutique de maintenir sa prétention à l'universalité? Lui permet-il de penser l'action, c'est-à-dire l'histoire et la politique?

Indem sie «Sein das verstanden werden kann» als «Sprache» begreift, scheint die philosophische Hermeneutik H.-G. Gadamers zwischen zwei Orientierungsmö: die eine, die der hegelschen Tradition verpflichtet ist, denkt die Sprache als Logos, d.h. als ein Vermittein, Mitteilen und Zirkulieren eines Sinnes. Die zweite denkt im gefolge Heideggers die Sprache als Poïesis, d.h. als ein Erschliessen des Seienden, als das sich Ereignen der Wahrheit. Man kann sich fragen, wie diese Antinomie in Wahrheit und Methode aufgehoben wird. Liegt aber in dieser Einheit der Schwerpunkt nicht auf Poïesis, insofern als das der Sprache inhärentes Spiel immer ein Wahrheits - und nie ein Freiheitsspiel ist? Kann aber eine Hermeneutik, die jeglichen Subjektivismus ablehnt, ihren Universalitätsanspruch aufrechterhalten? Vermag eine so konzipierte Hermeneutik, das Handeln, d.h. die Geschichte und die Politik überhaupt zu denken?

\section{AUTEUR}

\section{JEAN-MICHEL BUÉE}

Centre Eric Weil -L U.F.M. expérimentale de Lille 